\title{
Experiencias para desarrollar competencias de emprendimiento en la formación universitaria
}

Experiences to develop entrepreneurship competences in university education

Magdalena Emilia Ordóñez Gavilanes. ${ }^{1}$, Renán Teodoro Rodríguez Pillaga. ${ }^{2}$, Priscila Isabel Ruiz Alvarado. ${ }^{3}$

Recibido: 08-07-2019 / Revisado: 17-07-2019 /Aceptado: 13-08-2019/ Publicado: 10-09-2019

\begin{abstract}
.
DoI: https://doi.org/10.33262/cienciadigital.v3i3.4..890

There is no doubt that Higher Education can generate an impact on the economic development of a country and one of the ways in which it is possible to act is in the promotion and impulse of entrepreneurship as a key activity. This training process can be developed through the substantive functions of the university: Teaching, Research and Innovation and Linkage with Society. For this, the present work from the perspective of the teaching action, aims to publicize the entrepreneurial experiences of the students of the Business Administration Career of the Universidad Católica de Cuenca, who decided to face the challenge of undertaking, putting in Practice the knowledge acquired during your training process, under the direction of teaching entrepreneurs. In this qualitative study, the competencies are approached from the perspective of complex thinking, its scope is descriptive, it uses the technique of participant observation to extract results, which reflect the application of important competences inherent to entrepreneurship in the activities of the students of the Career
\end{abstract}

\footnotetext{
${ }^{1}$ Universidad Católica de Cuenca, Extensión Cañar, meordonez@ucacue.edu.ec

${ }^{2}$ Universidad Católica de Cuenca, Extensión Cañar, rtrodriguezp@ucacu.edu.ec.

${ }^{3}$ Universidad Católica de Cuenca, Extensión Cañar, pruiza@ucacue.edu.ec.
} 
under study, concluding that it is possible to develop these competences through curricular and extracurricular study activities.

Keywords: Entrepreneurship, university, competences, innovation

\section{Resumen}

Es indudable que la Educación Superior puede generar impacto en el desarrollo económico de un país y una de las formas en las cuales es posible actuar, es en el fomento e impulso al emprendimiento como actividad clave. Este proceso formativo puede ser desarrollado a través de las funciones sustantivas de la universidad: Docencia, Investigación e Innovación y Vinculación con la Sociedad. Para ello el presente trabajo desde la perspectiva de la acción docente, tiene como objetivo dar a conocer las experiencias de emprendimiento de los estudiantes de la Carrera de Administración de Empresas de la Universidad Católica de Cuenca, quienes decidieron enfrentar el reto de emprender, poniendo en práctica los conocimientos adquiridos durante su proceso de formación, bajo la dirección de docentes gestores de emprendimiento. En este estudio cualitativo se aborda las competencias desde el enfoque del pensamiento complejo, su alcance es descriptivo, utiliza la técnica de la observación participante para extraer resultados, los mismos que reflejan la aplicación de importantes competencias inherentes al emprendimiento en las actividades los estudiantes de la Carrera en estudio, concluyendo que es posible desarrollar estas competencias mediante actividades de estudio curriculares y extracurriculares.

Palabras Clave: Emprendimiento, universidad, competencias, innovación

\section{Introducción}

La educación debe ser una herramienta que permita a las personas adquirir conocimientos, pero sobre todo desarrollar competencias aplicables al contexto practico, es decir medios para enfrentar la vida laboral al momento de culminar los estudios superiores. Un estudiante debe estar en la posibilidad de desempeñarse con éxito en un empleo o generar su propia empresa, esto implica poder insertarse con éxito en el mercado laboral, para ello es necesaria una educación de calidad, que responda a las expectativas del entorno y sobre todo de solución a las problemáticas de la sociedad. 
Es entonces un compromiso ineludible de las instituciones de educación superior impulsar el emprendimiento a través de la enseñanza de herramientas teórico-prácticas que faciliten a los estudiantes el desarrollo de competencias relacionadas a este proceso.

Peter Drucker implícitamente en su obra La innovación y el empresariado innovador, reconoce la importancia de la enseñanza del emprendimiento, sobre todo en la universidad, manifestando que no existe personalidad emprendedora, y que es susceptible de ser formada, pues las habilidades del emprendedor se pueden aprender, implican una conducta, una actitud que hace que la persona sea capaz de intuir una oportunidad de negocio y poner en práctica acciones arduas, creativas, difíciles y arriesgadas, que dan como resultado la creación de una empresa o negocio. Siendo así, el emprendimiento puede ser considerado más como una conducta que como un rasgo del carácter y, por tanto, puede ser enseñable. (Drucker 1986).

El emprendimiento en el ámbito universitario es un proceso de aprendizaje que comprende un conjunto de actividades curriculares y extracurriculares, en las cuales la universidad incluye al estudiante y aprovecha las experiencias acumuladas, con el reto de fomentar una cultura emprendedora y un enfoque hacia la preparación para enfrentar el mundo que los rodea y propiciar el cambio social, político y estructural que se requiere.

Al respecto Cabello (2004), en su artículo "Emprendedurismo dentro del ámbito universitario" define al emprendedor universitario como: el ser humano que cuenta con estudios de nivel superior y el suficiente conocimiento y autoconocimiento para desarrollar su negocio propio , pero sobre todo está motivado para formular y desarrollar sus sueños, poniendo en práctica la innovación con criterio asertivo y proactivo en los escenarios económicos o sociales, en donde le sea posible gestionar, sobre la base de competencias que lo visualicen como un individuo especial, que por sus características propias, es eficaz y posee ese impulso para sobresalir de entre los demás, que lo lleva a producir impactos en la rama productiva de su elección.

Para Krauss (2011) sin duda alguna en la actualidad existe un consenso cada vez mayor sobre el rol de la universidad como formadora de emprendedores. No solo a nivel de Latinoamérica, 
se escucha la premisa "Fomentar la cultura emprendedora", que no es tarea simple, a nivel mundial se adoptado el emprendimiento como una actitud dinamizadora de la economía de un país.

En el caso del Ecuador, la efervescencia del emprendimiento está enraizada en la cultura misma de sus habitantes, razón por la cual esta temática es tratada desde diversos enfoques. El Ecuador es uno de los países que presenta mayor índice de actividad emprendedora a nivel de América Latina y el Caribe, con un porcentaje del 31.8\% lidera el listado de los países de la región (Global Entrepreneurship Monitor, 2016).

En este contexto el presente artículo tiene como objetivo exponer una síntesis de como las experiencias implícitas en la formación universitaria contribuyen a la generación de competencias de emprendimiento.

\section{Las competencias y el emprendimiento}

El término "competencia" es de reconocida importancia en la actualidad, desde un enfoque conductista bajo la perspectiva de la Dra. Martha Alles, en su libro Dirección Estratégica de Recursos Humanos, se refiere a las competencias como: (. . .) las características de personalidad, devenidas en comportamientos, que generan un desempeño exitoso en un puesto de trabajo (Alles, 2010).

Atendiendo a un enfoque de contingencias, una competencia puede ser definida como una característica implícita en un individuo que está causalmente relacionada a un estándar de efectividad y/o a una performance superior en un trabajo o situación (Spencer L. \& Spencer S, 1993).

Sin embargo, esta experiencia aborda la conceptualización de competencia desde un enfoque sistémico-complejo, priorizando la formación de personas integrales con compromiso ético, que busquen su autorrealización, que aporten al tejido social y que, además, sean profesionales idóneos y emprendedores (Tobón, 2008)

Bajo esta concepción una competencia representa: 
Procesos complejos de desempeño con idoneidad en determinados contextos, integrando diferentes saberes (saber ser, saber hacer, saber conocer y saber convivir), para realizar actividades y/o resolver problemas con sentido de reto, motivación, flexibilidad, creatividad, comprensión y emprendimiento, dentro de una perspectiva de procesamiento metacognitivo, mejoramiento continuo y compromiso ético, con la meta de contribuir al desarrollo personal, la construcción y afianzamiento del tejido social, la búsqueda continua del desarrollo económico-empresarial sostenible, y el cuidado y protección del ambiente y de las especies vivas (Tobón, 2008, p.5)

La aplicación de competencias tiene como meta producir un cambio, en el sistema educativo la meta es la formación de sujetos competentes. El mundo de las competencias se ha introducido en todas las ciencias y en las actividades humanas, razón por la que se han ocupado del tema tanto los docentes como los gestores del ámbito empresarial, las competencias son la base para la formación de emprendedores.

Mientras el concepto de emprendimiento como proceso, se lo relaciona con actividades vinculadas a la creación de empresas. El término "emprendimiento" tiene su origen semántico en la palabra francesa "entreprende", comprendida en ese entonces como "hacer algo" (Vásquez, 2017). Este concepto se convirtió en un descriptor de la capacidad humana para concretar resultados con eficiencia, eficacia y efectividad, lo que llevó a incorporar el término en la economía desde finales del siglo XVII en el tratado "Ensayo en la naturaleza general del comercio", del banquero parisino Richard Cantillón (Gama 2004).

Timmons (1999) concibe el emprendimiento como: "la capacidad para crear algo partiendo desde cero, complementada con la habilidad para conformar equipos y liderarlos resaltando sus destrezas; la capacidad para gestionar recursos y administrarlos y la pericia para asumir riesgos calculados" (p.62).

Según el Centro de Emprendedurismo de la Universidad de Miami, citado por la investigadora venezolana Cabello (2004) emprender es el proceso de identificar, desarrollar y dar vida a una visión, que puede ser una idea novedosa, una oportunidad o simplemente una mejor manera de hacer las cosas; y cuyo resultado final es la creación de una nueva empresa, formada bajo condiciones de riesgo y considerable incertidumbre. 
Cuando se hace referencia a la persona que emprende, Schumpeter (1934) conceptualiza el término "emprendedor" haciendo énfasis en la innovación desde el punto de vista de romper con lo tradicional y el liderazgo con talento especial para identificar el mejor modo de actuar. Pudiendo entonces establecer que desde esa época ya se atribuye al emprendedor, competencias que lo convierten en un tipo especial de persona, con la habilidad de ver las cosas como nadie más las ve.

Entonces, es pertinente expresar que "la competencia es inherente al emprendimiento y se construye a partir de la interacción del individuo con los entornos" (Ahumada, 2013, p.44). Al parecer la experiencia permite emitir este juicio por demás acertado. La interacción de los individuos, en este caso estudiantes, con su entorno inmediato permite establecer un vínculo con la sociedad y sus problemas, a partir de los cuales surge el interés de solución de los mismos dando paso a la aventura de emprender. Si bien la teoría nos encamina a comprender que la decisión de emprender guarda estrecha relación con la necesidad de generar ingresos

para satisfacer las necesidades y en otros casos la oportunidad de un negocio propio, la actividad emprendedora en la universidad se puede visualizar como un acercamiento entre el estudiante y la problemática social que impulsa a crear e innovar con actitud proactiva para plasmarlas en experiencias prácticas de negocio.

\section{El emprendimiento como eje transversal en la educación universitaria}

Desde el enfoque del pensamiento complejo la Universidad Católica de Cuenca, busca la formación de seres humanos integrales, como expresa su filosofía institucional, con ética de vida y espíritu emprendedor, reflejado en competencias necesarias para su realización personal, compromiso con el desarrollo del tejido social y productivo, con un desempeño profesional empresarial que considera el desarrollo con sostenibilidad y el cuidado del medio ambiente.

Para Vásquez (2017) la universidad debe plantear y hacer posible que sus educandos, de manera transversal, en su proceso formativo adquieran las necesarias y suficientes destrezas y competencias que permitan convertirse en profesionales visionarios que viabilicen la creación de nuevas oportunidades y empresas. 
Para ello las buenas prácticas de emprendimiento son parte de la educación diaria de los estudiantes en la Universidad Católica de Cuenca, procurando que en cada una de las asignaturas se promueva el tema como un eje transversal del aprendizaje. El objetivo es impulsar un modelo educativo que tome en cuenta el desarrollo de competencias emprendedoras, el mejoramiento de la preparación de los jóvenes, para lograr una mayor pertinencia y habilidades para incorporarse al mundo laboral, permitiendo generar un crecimiento económico real en la sociedad donde se desarrollan.

En el ámbito educativo se debe reconocer la importancia que tiene contar con esquemas de educación para el emprendimiento, alineados desde la estrategia hasta la conformación del diseño curricular.

Es así que las buenas prácticas para mejorar las competencias de emprendimiento en la Carrera de Administración de Empresas se las puede resumir en las siguientes acciones:

- En el diseño curricular, consta la materia de Emprendimiento, Liderazgo y Simulación de negocios.

- Los contenidos de enseñanza en las asignaturas propenden a la realización de planes de negocio para mejorar la realidad y contribuir al desarrollo del tejido productivo local.

- Los estudiantes desarrollan investigaciones innovadoras, para generar productos con materias primas propias de la región, sobre la base de conocimientos ancestrales.

- Se realizan eventos y ferias de emprendimiento, con la participación de estudiantes y docentes, espacio en donde se plantean proyectos auspiciados por empresas públicas y privadas.

- Los estudiantes comparten experiencias con emprendedores locales en el Centro de Emprendimiento de la universidad, en donde se brinda asesoría en gestión empresarial a MIPYMES.

Sobre la base de lo expuesto, se puede decir que el trabajo de formación en emprendimiento implica el desarrollo de una serie de actividades curriculares y extracurriculares, guiadas por docentes. 


\section{Experiencias de emprendimiento}

Una experiencia en la formación universitaria puede enmarcarse en el concepto de Dewey (2004) que la describe como la totalidad de las relaciones del individuo con el medio ambiente. Se trata de una unidad de análisis que toma en consideración las interacciones entre las personas y el contexto, las cuales se determinan mutuamente, esto es, tienen un carácter sistémico transaccional.

La generación de estas experiencias propicia una triada: universidad - estudiante emprendimiento, es decir, una interacción de expectativas que justifican su interés mutuo. La universidad, como factor clave del desarrollo social, tiene un compromiso ineludible con la sociedad, a la cual debe entregar como resultado talento humano especializado, competente, capaz de transformar la matriz productiva y por lo tanto la economía nacional; por su parte el estudiante, como actor clave del proceso, encuentra su inspiración y motivación para emprender en las aulas universitarias con el apoyo de los docentes y finalmente el fomento de un tipo de emprendimiento innovador, sostenible y responsable con la sociedad.

Bajo este contexto, se puede decir que afianzar conocimientos y motivar a los estudiantes es la aspiración de los docentes, sin embargo, impulsarlos en busca de una aventura empresarial requiere de ciertas acciones, condiciones y escenarios que deben ser facilitados para propiciar el desarrollo del emprendimiento.

En la Universidad Católica de Cuenca, Extensión Cañar, se han desarrollado actividades de emprendimiento desde hace muchos años, socializadas en exposiciones o ferias académicas, que son espacios en donde se dan a conocer proyectos de investigación elaborados por los estudiantes, con el apoyo de docentes de las diferentes asignaturas y Unidades Académicas de la universidad.

En este acercamiento al emprendimiento los educandos desarrollan competencias, más aún en el caso de la Carrera de Administración de Empresas, donde los estudiantes aprovechan estas experiencias para traducirlas en trabajos de investigación previo a su graduación.

El escenario de la experiencia de innovación que se presenta en este artículo, es la Universidad Católica de Cuenca, Extensión Universitaria de Cañar, fundada hace 25 años, 
en la ciudad del mismo nombre, perteneciente a la Provincia de Cañar - Ecuador, en la cual se realizó el evento denominado "Plaza de Emprendimientos", actividad anual desarrollada en cooperación con instituciones tanto del sector público, como del sector privado que fomentan el emprendimiento a nivel local.

Los objetivos del evento fueron los que a continuación se detallan:

- Generar un espacio de diálogo de saberes en el ámbito del emprendimiento entre los miembros de la Comunidad Universitaria.

- Impulsar a los estudiantes de las diferentes Unidades Académicas a participar activamente mediante la generación y exposición de proyectos de emprendimiento de manera grupal e individual.

- Establecer alianzas estratégicas con instituciones públicas y privadas que apoyen la gestión del proyecto, sobre la base del criterio de responsabilidad social.

En función de estos objetivos, los docentes gestores de emprendimiento plantearon estrategias, que propiciaron experiencias enriquecedoras para los estudiantes, válidas en el desarrollo de competencias inherentes al emprendimiento.

\section{Metodología}

Se presentan experiencias de formación universitaria bajo el paradigma del pensamiento complejo. Este estudio cualitativo, de alcance descriptivo, utilizó la técnica de la observación participante para acceder con facilidad y eficiencia a las experiencias vividas por los estudiantes, permitiendo interactuar con ellos durante todo el proceso.

En el evento Plaza de Emprendimientos, analizado en esta investigación, participaron con ideas de negocio la totalidad de las Carreras de la Extensión Universitaria de Cañar. La exposición reunió a 600 estudiantes, agrupados en 31 proyectos innovadores, de los cuales se seleccionaron tres emprendimientos pertenecientes a la Carrera de Administración de Empresas, que obtuvieron las puntuaciones más altas de acuerdo a los siguientes parámetros que sirvieron de base para la convocatoria: 
- Creatividad e Innovación en la idea de negocio.

- Factibilidad de la idea de negocio

- Trabajo en Equipo.

- Gestión de comercialización.

Esta investigación de campo presenta un estudio estructurados en tres etapas:

\section{Experiencias ex-ante el evento}

Los estudiantes, previo al evento, desarrollaron actividades que sirvieron de sustento para los proyectos que se presentaron en la Plaza de Emprendimientos. Se realizaron estudios de mercado con el objetivo de conocer los gustos y preferencias de los consumidores, considerando que actualmente su comportamiento presenta una tendencia hacia los productos de procedencia natural que contienen altos valores de nutrientes para cuidar la salud y alargar en lo que se pueda el promedio de vida de las personas. También se llevaron a cabo análisis de factibilidad para identificar las posibilidades de éxito o fracaso de los proyectos.

Los productos ofertados por los estudiantes obedecieron a una segmentación de mercado bien definida, para ello se diseñó un plan de marketing que permitió establecer estrategias pertinentes al microentorno y macroentorno del emprendimiento, que permitan obtener rentabilidad y establecer una ventaja competitiva sostenible, a través de un producto que posee un valor agregado percibido por el consumidor.

\section{Experiencias durante el evento}

Los emprendimientos seleccionados que involucran estudiantes de la Carrera de administración de empresas, adoptaron el slogan "Aliméntate de verdad innovando lo nuestro", en él intervinieron 50 estudiantes, en un porcentaje del $77 \%$ de mujeres y el $33 \%$ de hombres, distribuidos en equipos de trabajo, con asignaciones de tareas específicas, bajo la coordinación de seis docentes gestores de emprendimiento. Su objetivo principal fue producir y comercializar productos sobre la base de criterios artesanales y técnicos, con alimentos saludables propios de la zona, cuyas características nutricionales fueron aprovechadas para satisfacer las necesidades alimenticias y las expectativas de las personas 
que desean mejorar su salud con calidad, incentivando el consumo de productos de la zona, para generar nuevas fuentes de empleo y aprovechar las zonas agrícolas del cantón Cañar que hoy se encuentran abandonadas por causa de la migración tanto al interior del país como al extranjero.

\section{Experiencias ex-post}

Son las actividades de retroalimentación o feedback, que se realizaron a través de talleres con la participación de estudiantes y docentes gestores de emprendimiento. En este espacio se propició un intercambio de experiencias que permitió obtener información válida que sirvió a los docentes de sustento a las observaciones realizadas en etapas anteriores, determinando con mayor certeza las competencias que se pusieron en práctica antes, durante y después de las experiencias de emprendimiento.

\section{Resultados}

Se puede resumir el balance formativo de estos proyectos, en experiencias que ponen en manifiesto ciertas actitudes y competencias específicas que apoyan el proceso de emprendimiento desplegadas por los estudiantes antes, durante y después del evento y que pudieron ser observadas por los docentes responsables de los proyectos, entre ellas, se destacaron:

- El sentido de pertenencia y responsabilidad social; demostrada en el momento de seleccionar alimentos propios de la zona para la elaboración de productos, cuyo cultivo aporta al desarrollo del tejido productivo local.

- La identificación de oportunidades; indagando y descubriendo en los cultivos andinos, la fuente de satisfacción de necesidades presentes en la población.

- La capacidad de traducir una idea en un plan de acción; fueron capaces de convertir las estrategias de su plan de negocios en tácticas planeadas con eficiencia y eficacia, es decir concretar la idea de negocio.

- El compromiso y confianza en sí mismo; mostrándose optimistas y seguros de conseguir la finalidad propuesta. 
- La ética y responsabilidad por el trabajo; para asumir el reto de emprender y cumplir a cabalidad las obligaciones a ellos encomendadas, con el fin de aportar a la consecución de la misión y visión del emprendimiento.

- El trabajo en equipo; demostrando su capacidad para colaborar y ayudar a otros compañeros en las actividades requeridas.

- El empoderamiento; enfrentando y definiendo responsabilidades.

- La perseverancia y constancia en el cumplimiento de metas; firmes ante la posibilidad de abandonar el proyecto, pese a la carencia de ciertos recursos y a la complejidad de los procesos que implicaban su realización.

- La organización como equipo de trabajo auto dirigido; la lucha por la calidad del trabajo, aplicando con efectividad los principios de calidad aprendidos en las aulas universitarias.

- La orientación al cliente; demostrando el afán de contribuir a la satisfacción de las necesidades de la gente.

\section{Discusión}

Los estudiantes buscaron, a través de la innovación, dar respuesta en forma efectiva a carencias en el ámbito de la salud y la nutrición; demostraron iniciativa, creatividad y pensamiento analítico. Idearon nuevas soluciones frente a necesidades, presentando proyectos novedosos y originales a la medida de las aspiraciones del mercado meta, reconociendo las causas y las consecuencias de las acciones; manifestaron liderazgo y orientación a los resultados.

El equipo de estudiantes administró correctamente los procesos de trabajo, poniendo énfasis en realizar correctamente las actividades; fue destacada la comunicación grupal, como competencia impulsora del dialogo de saberes y experiencias para establecer soluciones y consensos, la misma que se vio manifiesta en el equipo de trabajo y permitió la comprensión de información y desarrollo de las interrelaciones personales.

Se contribuyó con la investigación formativa pues se desarrollaron actividades investigativas directamente relacionadas con el emprendimiento, es decir las actividades que implican 
emprender en forma segura y sostenible, demostrando que han atravesado la barrera del SABER al SABER HACER.

Este estudio presenta resultados que podrían ser analizados a profundidad a través de investigaciones que permitan mejorar los procesos pedagógicos de enseñanza aprendizaje, para generar competencias no solamente en el campo del emprendimiento.

\section{Consideraciones finales}

Las competencias descritas están inmersas en el perfil de egreso de los estudiantes de la Carrera de Administración de Empresas de la Universidad Católica de Cuenca, Extensión Cañar. En consecuencia, esta experiencia innovadora que obtuvo los primeros lugares en el proyecto Plaza de Emprendimientos, generó competencias que ayudarán al futuro profesional a enfrentar los desafíos que le impone el entorno, frente al cambio de la matriz productiva en el Ecuador que exige talento humano preparado, que asuma los riesgos de la innovación en un ambiente de incertidumbre.

Las actividades curriculares y extracurriculares que fomenten la cultura del emprendimiento en los estudiantes y que contribuyan a desarrollar la creatividad e innovación, se constituyen en experiencias que permiten concretar los aprendizajes impartidos en el aula de clase y hacerlos significativos y aplicables a la realidad, sobre todo en escenarios actuales de cambio, en donde el talento humano juega el papel protagónico para el desarrollo de la sociedad, es por ello que se recomienda fomentar en mayor grado actividades de emprendimiento bajo la orientación de docentes con participación activa de los estudiantes.

\section{Referencias Bibliográficas}

Ahumada, L. (2013). Las competencias aplicadas al emprendimiento. Revista Escenarios, 11(1), 44-66. Recuperado de file:///C:/Users/Usuario\%20MC/Downloads/DialnetActitudesEmprendedorasDeLosEstudiantesUniversitari-3797740.pdf

Alles, M. (2010). Dirección Estratégica de Recursos Humanos: Gestión por competencias. Argentina: Editorial Gránica. 
Cabello, C. (2004). Emprendedurismo dentro del ámbito universitario. Recuperado de https://www.mific.gob.ni/Portals/0/Portal\%20Empresarial/u16.\%20Emprendedurismo\%20e n\%20el\%20\%C3\%A1mbito\%20universitario.pdf

Dewey, J. (2004). Experiencia y Educación. Madrid, España: Editorial Biblioteca Nueva.

Drucker, P. (1986). La innovación y el empresariado innovador: la práctica y los principios. Buenos Aires, Argentina: Editorial Norma.

Escuela Superior Politécnica del Litoral [ESPOL] y Graduate School of Management [ESPAE]. (2017). Global Entrepreneurship Monitor, Ecuador-2016. Recuperado de http://www.espae.espol.edu.ec/wp-content/uploads/2017/06/ReporteGEM2016.pdf

Gama, S. (2004). Pedagogía activa del emprendimiento para el desarrollo económico local: el caso del colegio Juan Rulfo de USME, Bogotá. Tesis de Maestría en Planificación y Administración del Desarrollo Regional. Universidad de los Andes. Bogotá, Colombia.

Krauss, C. (2011). Actitudes emprendedoras de los estudiantes universitarios: $\quad$ El caso de la Universidad Católica de Uruguay. Recuperado de file:///C:/Users/Usuario\%20MC/Downloads/DialnetActitudesEmprendedorasDeLosEstudiantesUniversitari-3797740.pdf

Schumpeter, J. (1934). The Theory of Economic Development. United States: Cambridge, MA, Harvard University Press.

Spencer, L. y Spencer, S. (1993). Competence at work: Models for Superior Performance. United States: Ed. Jhon Wiley \& Sons.

Timmons, J. (1999). New Venture Creation: Entrepeneurship for the 21 Century. Boston, United States: Mc Graw-Hill Higher Education.

Tobón, S. (2008). La formación basada en competencias en la educación superior: El enfoque complejo. Recuperado de: http://cmapspublic3.ihmc.us/rid=1LVT9TXFX$\underline{1 \mathrm{VKC} 0 \mathrm{TM}-}$ 
16YT/Formaci\%C3\%B3n\%20basada\%20en\%20competencias\%20(Sergio\%20Tob\%C3\%B

$\underline{3 n) . p d f}$

Vásquez, C. (2017). Educación para el emprendimiento en la Universidad. Estudios de la Gestión, 121-147. Recuperado de http://repositorio.uasb.edu.ec/bitstream/10644/5957/1/07-

ES-Vasquez.pdf 
PARA CITAR EL ARTÍCULO INDEXADO.

Ordóñez Gavilanes, M., Rodríguez Pillaga, R., \& Ruiz Alvarado, P. (2019). Experiencias para desarrollar competencias de emprendimiento en la formación universitaria. Ciencia Digital, 3(3.4.), 365-380. https://doi.org/10.33262/cienciadigital.v3i3.4.890

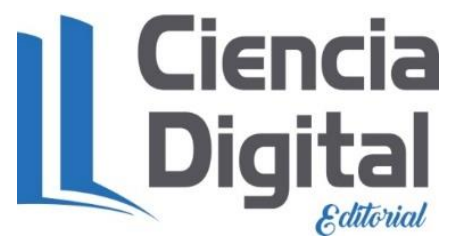

El artículo que se publica es de exclusiva responsabilidad de los autores y no necesariamente reflejan el pensamiento de la Revista Ciencia Digital.

El artículo queda en propiedad de la revista y, por tanto, su publicación parcial y/o total en otro medio tiene que ser autorizado por el director de la Revista Ciencia Digital.
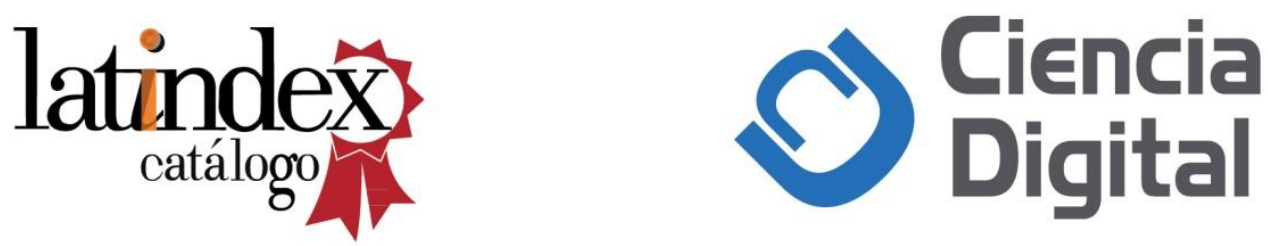